\title{
Designing elicitation of expert knowledge into conditional probability tables in Bayesian networks: choosing scenario
}

\author{
I. Alkhairy ${ }^{\mathrm{ab}}$ and S. Low-Choy ${ }^{\mathrm{cd}}$

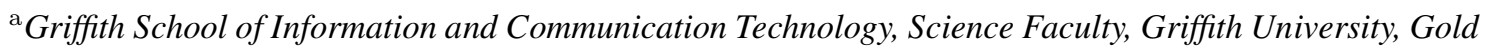 \\ Coast Campus, QLD 4222, Australia \\ ${ }^{\mathrm{b}}$ Department of Mathematics, Al Qunfudhah University College, Umm Al Qura University, Makkah 24382, \\ Saudi Arabia. \\ ${ }^{\mathrm{C}}$ Office of the Dean, Arts / Education / Law Group, Griffith University, Mt Gravatt Campus, QLD \\ 4122, Australia. \\ ${ }^{\mathrm{d}}$ Researcher Education and Development unit, Office of Research, Griffith University, Nathan Campus, QLD \\ 4111, Australia. \\ Email: ibrahim.alkhairy@griffithuni.edu.au
}

\begin{abstract}
For many researchers there is increasing pressure to collect and analyze bigger datasets, from sources such as analytics, online surveys or spatial datasets. Bayesian networks (BNs) provide a feasible and intuitive means of developing explanatory models with diverse stakeholders, having limited quantitative expertise. However, when a large number of variables and levels are involved as potential inputs to a BN, the more resources are required to evaluate alternative models. Our motivation is to design a large species distribution modeling (SDM) experiment, in the Biodiversity and Climate Change Virtual Laboratory. We show how BNs, elicited from experts, can be used to inform design of these kinds of large computing experiments.

In this context we examine how settings of some SDM algorithms potentially affect the quality of the prediction. For example, one setting could be the choice of covariates used as input to the SDM, with three levels: a minimal, an extensive set or something in between. A conditional probability table (CPT) quantifies the child node (e.g. quality of prediction) as it depends conditionally on each of the parents (here settings). Guidelines on eliciting CPTs generally advise modellers to simplify the elicitation task by keeping to a minimum the number of parent nodes and parent/child states. The literature on BNs indicates that elicitation of more complex CPTs may be too demanding for experts, because of the time required.
\end{abstract}

In the context of large CPTs, an often encountered problem is the sheer amount of information asked of the expert (number of scenarios). Here we propose that an elicitation strategy can be designed according to statistical criteria: to ensure adequate coverage of the CPTs, in an efficient manner, to make best use of the scarce resources like the valuable time of the experts. This is essentially a problem of experimental design.

Some software tools such as CPT calculator support specification of large CPTs, but implicitly adopt a particular kind of experiment design. Here we conduct experiments to evaluate designs for eliciting expert knowledge to help quantify CPTs that define B Ns. We consider three types of design of elicitation: Taguchi as a kind of screening design, CPT calculator's design, and a composite. In the case study, we asked modellers to consider how different settings affect the quality of an algorithm used to construct a SDM. Limiting the number of scenarios avoids tiring the experts, which can lead to inaccuracies. Eliciting and encoding CPTs was examined using a model-based "outside-in" Elicitator approach to quantitative elicitation, which allows experts to specify their opinions with uncertainty.

Our results determined that the most important settings with the largest positive impacts on the quality of prediction were: the choice of real absence data, quadratic complexity of the function and the choice of the expert's minimal subset of variables. In addition, there were differences arising due to the choice of design and the elicitation scenarios. Overall, we found that the Taguchi OA design was more efficient because the effect sizes estimated for CPT calculator had more uncertainties than for the Taguchi design.

Keywords: Bayesian networks, conditional probability tables, elicitation of expert knowledge, design of elicitation, species distribution model 


\section{INTRODUCTION}

Species distribution models (SDMs) are techniques that produce a statistical model for the relationship between a species and its environment, forming an ecological niche model (Elith and Leathwick, 2009). These models are using computer algorithms to predict the distribution of species in geographic space on the basis of data (Reiss et al., 2011). There is an extensive literature that compares SDM algorithms, but does not necessarily consider the sensitivity to settings. Here, we use this case study to demonstrate how a Bayesian network can be used to design a large sensitivity study, and in particular conduct a "risk assessment" to choose which settings should be investigated.

BNs represent the model using a conditional probability graph comprising several nodes, and arrows linking nodes. Underlying each link is the specification of a conditional probability distribution, where one or more parent nodes are related to a child node, as indicated by a link (Korb and Nicholson, 2010). For instance, Figure 1 is a very simple BN involving four parent nodes with one child node, and shows how the most important settings of a SDM algorithm, a generalized linear model (GLM), potentially affect the quality of the results. The nodes are typically discretized into a number of levels. Here, each node has 3 levels (Table 1).

Table 1. Four settings of GLM each have three levels

\begin{tabular}{|c|c|c|c|c|c|}
\hline & Level & Covariates & Choice of absences & Complexity of regression & Link function \\
\hline Best case & 2 & Minimal (M) & Real (R) & Quadratic (Q) & Cauchit (C) \\
\hline & 1 & Between (B) & Pseudo Stratified (S) & 2-way interactions (2) & Gompertz (G) \\
\hline Worst case & 0 & Extensive (E) & Pseudo CRD (P) & Main effects (F) & Logit (L) \\
\hline
\end{tabular}

A conditional probability table (CPT) quantifies this relationship between parents and child node (Korb and Nicholson, 2010). Generally, these nodes and their conditional parameters $\theta$ together define the CPTs such that $\theta=\operatorname{Pr}(y \mid$ parents $(y))$, where $y$ is the child node. Here in our case (Figure 1), $\theta_{i j k l}=\operatorname{Pr}\left(Q o P_{i j k l} \mid C o v=\right.$ $i, C o m=j, C h o=k, \operatorname{Lin}=l)$, where Cov, Com, Cho and Lin are parents of the child node QoP; $\theta_{i j k l}$ comprises the conditional probabilities; $i=0,1, \ldots, I-1$ is the $i$ th level of $C o v, j=0,1, \ldots, J-1$ is the $j$ th level of $C o m$ and $k=0,1, \ldots, K-1$ is the $k$ th level of $C h o$ and $l=0,1, \ldots, L-1$ is the $l$ th level of Lin .

In the context of BNs, the goal of expert elicitation is to discover stet probability distribution $p(\theta)$ (Grigore et al., 2013) which can adequately represent the experts' opinion and their uncertainty regarding the CPTs. However, when there are a large number of scenarios in the CPT, it is a challenge to ask experts about each scenarios. In any event, the elicitation strategy must be based on a proper experimental design (Box et al., 2005).

This paper will focus on the tailoring of classical design of experiments to choose some scenarios (Section 2.1). Then, we will examine the methodology of eliciting the CPTs using the Elicitator method, and encoding a plausible range of values (Section 2.2). Finally, the expert's knowledge is used to determine which SDM algorithm settings of GLM have largest impact on quality of prediction (Section 3).

\section{Method}

\subsection{Design of elicitation}

Statistical experimental design, together with the basic ideas underlying DOE, was developed in the early 1920s from the work of Sir Ronald Aylmer Fisher (Fisher, 1960). In the context of CPTs, which scenarios are asked could be selected by using principles from the design of experiments, which can help choose questions strategically both to minimise the number of questions and to cover the area of interest Box et al. (2005). 
Fully factorial design. It is ideal if a fully factorial experiment can be conducted, as all of the data is present for each node and level, i.e. it consists of all possible combinations of levels for all factors (Antony, 2003). The total number of parameters (the dimension of $\theta$ ) defines the minimum number of questions for a fully factorial designed CPT and can be donated as $N=I \times J \times K \times L$. In our case study, each node has three levels (so, $I=J=K=L=3$ ) and hence, the total number of scenarios would require $3 \times 3 \times 3 \times 3=81$ questions. However, it is also important to limit the number of scenarios to avoid tiring the experts, which can lead to inaccuracies, especially here for complex CPTs (Marcot et al., 2006). Here, note how many scenarios an expert could answer in one setting. This paper will consider three types of design of elicitation: a Taguchi Orthogonal array design, CPT calculator design, and a composite.

CPT calculator design . CPT calculator is a tool proposed by Cain (2001) that supports experts to quantify the CPTs that define BNs. This means that scenarios are selected using a one-at-a-time design, where each scenario changes just one parent level at a time i.e. CPTs are specified by extrapolating from the best and the nearly best scenario and worst scenarios (Cain, 2001; Antony, 2003). CPT calculator adopts a simple encoding model to interpolate the CPT from questions about several scenarios. Linear interpolation is used to interpolate other scenarios using an interpolation factor (IF). Each IF is calculated for each transition between best case and changing just one setting. They are all calculated in relation to the difference between the highest probability for the best case and the lowest one (when all parents are in the worst case). For example, the interpolation factor for parent Cov changing from best to worst is $I F_{1}=\left(\theta_{E R Q C}-\theta_{E P F L} /\left(\theta_{E R Q C}-\theta_{E P F L}\right)\right.$. The indices ERQCPFL are explained in Table 1. For more detail on these interpolations, see Appendix 2 from Cain (2001).

In our case study (Table 1), $\theta_{M R Q C}$ refers to the quality of prediction in the best case, $\mathrm{M}$ indicates the expert chooses a minimal subset of covariate, $\mathrm{R}$ is the choice of real data, $\mathrm{Q}$ is quadratic complexity and $\mathrm{C}$ is the cauchit function. Then $\theta_{E P F L}$ refers to the quality of prediction in the worst case. There are many CPs that the expert did not have time to provide and need to be estimated, e.g. the quality of prediction $\theta_{E P Q C}$. To calculate these, we perform linear interpolation by multiplying $\theta_{M P Q C}$ (scaled by the lowest quality of prediction in the worst case $\theta_{E P F L}$ ) by the interpolation factor that is associated with the covariates parent changing from best to worst case $\left(I F_{1}\right)$. We use the equations like: $\left[\left(\theta_{i-1 j k l}-\theta_{1111}\right) \times I F_{1}\right]+\theta_{1111}$, where here $\theta_{1111}=\theta_{E P F L}$ refers to the worst case.

Taguchi's Orthogonal Arrays design . Taguchi's Orthogonal Array design is a classical type of fractional factorial design (Kacker et al., 1991) and based on the design matrix proposed by Taguchi and Konishi (1987). An orthogonal array (OA) of $h$ levels (more particularly a fixed level orthogonal array) can be denoted as $O A_{\widetilde{N}}\left(h^{m}\right)$ and is defined as an $\widetilde{N} \times m$ matrix, where $\widetilde{N}$ is the minimum number of scenarios and $m$ the number of nodes (settings). The common feature of OA is that each pair of parents has the same number of possible levels (for more detail, see Kacker et al., 1991). Taguchi OA provides an effective way to consider a large number of nodes in a minimum number of scenarios (Jugulum et al., 2004), which could give the full information of all parents (e.g. GLM settings) that affect the child node (i.e. quality of prediction). Performing a Taguchi's OA design first can save time particularly for expert, allowing honing of the key settings which give the highest response (Antony, 2003). To our knowledge, Taguchi OAs have not been considered in the context of expert elicitation, including of CPTs in BNs. Here, we will use this design to minimise the number of scenarios used to encode the CPT. The main drawbacks of this design that is there is no continuity between scenarios, which may make it hard for experts to elicit the probabilities. Taguchi's OA designs are always balanced to ensure that all levels from all parents should occur in an equal number of scenarios (Jugulum et al., 2004).

When designing the elicitation of CPTs, there are many catalogs of Taguchi OA (for more detail, see Kacker et al., 1991). Here, we use the three-level orthogonal arrays of $3^{s}$ for $s=2,3$ and 4 , where $s$ indexes the basic columns (parents) in constructing Taguchi OA. The symbols $(0,1,2)$ are used to denote the levels of each node (e.g. Table 1). The columns correspond here to the settings Cov, Cho, Com and Lin, while the elements of each column defines the level of each setting. In particular, the number of scenarios $\widetilde{N}$ of $O A_{\widetilde{N}}\left(h^{m}\right)$ can be chosen as a subset of all scenarios of a fully factorial design $h^{m}$. This means that $O A_{\widetilde{N}}\left(h^{m}\right)$ is defined as a $\tilde{N} / h^{m}$ fraction of a $h^{m}$. For example, we can use just nine scenarios of $O A_{9}\left(3^{4}\right)$, which is defined as a $9 / 3^{4}=1 / 9$ fraction of $3^{4}=81$ scenarios.

There are three steps to conduct the design of elicitation for a CPT using a Taguchi's OA design, where a complete three level OA with $3^{s}$ rows has $\left(3^{s}-1\right) /(3-1)$ columns. The first step is to write the basic columns $s$ determined by the number of columns $1,2,5,14, \ldots,\left(3^{s-1}-1\right) /(3-1)+1$, which would provide 
a complete factorial in $s$ basic factors. The most common format of Taguchi's OA design makes the inputs of the leftmost columns change less frequently than the rightmost columns. The next step is to generate the rest of columns using the generator $b_{1} x_{1}+b_{2} x_{2}+\ldots, b_{s} x_{s}$, where $x_{1}, \ldots, x_{s}$ are the basic columns and $b_{1}, \ldots, b_{s}$ are the coefficients of a specific factor. Finally, we compute the inputs for the rest of the columns, using the inputs of $s$ basic columns. For example, Table 2 shows the construction of the Taguchi $O A_{9}\left(3^{4}\right)$ design, where $\widetilde{N}=9=3^{2}$, hence requiring $s=2$ basic columns. Note that all levels from the generators are calculated using modulo 3 arithmetic (because each setting has 3 levels) such that an integer larger than or equal to three is replaced with its remainder after division by three. For example, for the last row (Cov=2, Cho=2) the generator $x_{1}+x_{2}|3=2+2| 3=1$ and $2 x_{1}+x_{2}|3=4+2| 3=0$. In R programming, we can use the DoE. base package to create a Taguchi OA design using the oa. design function (Groemping et al., 2017).

Table 2. Construction of four factors, which each have three levels using Taguchi's three level orthogonal arrays design including the generators of other factors.

\begin{tabular}{ccccc} 
& \multicolumn{4}{c}{ Factors (columns) } \\
\hline No. of Scenarios & Cov & Cho & Com & Lin \\
\hline $\mathbf{1}$ & 0 & 0 & 0 & 0 \\
$\mathbf{2}$ & 0 & 1 & 1 & 1 \\
$\mathbf{3}$ & 0 & 2 & 2 & 2 \\
$\mathbf{4}$ & 1 & 0 & 1 & 2 \\
$\mathbf{5}$ & 1 & 1 & 2 & 0 \\
$\mathbf{6}$ & 1 & 2 & 0 & 1 \\
$\mathbf{7}$ & 2 & 0 & 2 & 1 \\
$\mathbf{8}$ & 2 & 1 & 0 & 2 \\
$\mathbf{9}$ & 2 & 2 & 1 & 0 \\
\hline Generators $\Longrightarrow$ & $x_{1}$ & $x_{2}$ & $x_{1}+x_{2}$ & $2 x_{1}+x_{2}$
\end{tabular}

Composite of CPT calculator and screening designs. This design comes from just combining scenarios of both the Taguchi's OA and the CPT calculator designs. This means that the information is doubled, but it also provides a balance between the ease of the CPT calculator design (e.g. as "warm-up") and the efficiency of the Taguchi OA design.

\subsection{Eliciting CPTs with their uncertainties}

There is a main challenge, on how to present the expert with focused but easy to understand questions that can provide adequate information (Low Choy et al., 2009). This defining feature of the elicitation protocoldictates how the CPT parameters $\theta=\left\{\theta_{l m n ; i j k}\right\}$ are elicited. This includes the order, the wording and the framing which affects the cognitive processes of the experts and hence how they understand what is required (Low Choy et al., 2009). These issues are typically not discussed in BN literature. For instance, question wording, as used to elicit $\theta$ components, has been provided in few studies where CPTs were elicited (Pike, 2004 , is an exception).

Outside-in method (Elicitator method). "Outside-in" is an indirect approach for eliciting probabilities with uncertainty and was implemented by Low Choy et al. (2010) to quantify expert opinions about various scenarios. It asks for similar questions to the "Four-point" approach (Speirs-Bridge et al., 2010), but in a different order, with different statistical meaning. For Outside-in, the order of the questions ensures uncertainty is about the quality of prediction, whereas Four-point addresses uncertainty in the mean quality of prediction. Four pieces of information are asked in the Outside-in method. This would involve questions like: "We are going to think about 100 GLMs. Now consider some specific SDM settings defined by a specific set of covariates, choice of absences, complexity of regression and link function. We want you to tell us what is the smallest and largest number so that you're pretty certain, e.g. $80 \%$ or $90 \%$ or $95 \%$ sure, that the quality of prediction falls between these bounds? If you fit 100 GLMs with these settings, how many would have the quality between lower and upper quality you gave? What is the quality of prediction for SDMs in the best case, where the expert chooses a minimal subset of covariate, choice of real data, quadratic complexity of function and the cauchit function ..."?.

Encoding plausible range of values. The main goal of this way is to transfer the information elicited from the experts into statistical distributions that reflect the best estimates and their uncertainties (Low Choy et al., 
2009). Suppose that $Y_{i}$ refers to observations on quality of prediction for each scenarios defined by SDM settings $X_{1}, \ldots, X_{J}$. Beta regression can be used to reveal the expert's mental: estimate the influence of each setting on the quality of prediction, using information on the estimated quality of prediction for each scenario $i=1, \ldots, n$ where $Y_{i} \sim \operatorname{Beta}\left(\alpha_{i}, \gamma_{i}\right), \operatorname{logit}\left(\mu_{i}\right)=\beta_{0}+\sum_{j=1}^{J} X_{i j} \beta_{i j}$ and $\mu_{i}=\alpha_{i} /\left(\alpha_{i}+\gamma_{i}\right)$, where $\mu_{i}$ is the mean, $\alpha_{i}$ and $\gamma_{i}$ describe the expert's uncertainty about scenario. Using the Elicitator method, the most important objective is to ask expert about the mode (their best estimates) on a specific scenario. Mathematically, the expert's best estimate $m_{i}$ of the quality of prediction $Y_{i}$ is simply related to the Beta parameters and denoted as $m=\left(\alpha_{i}-1\right) /\left(\alpha_{i}+\gamma_{i}-2\right)$. The challenge is that for each scenario a single $Y_{i}$ is registered. For $i=1, \ldots, n$ scenarios, there is a distribution $p\left(Y_{i} \mid X_{i}, \alpha_{i}, \gamma_{i}\right)$ that reflects the expert's uncertainty.

In many commercial software packages, beta regression is not allowed to have degrees of freedom $\nu_{i}=\alpha_{i}+\gamma_{i}$ changing over data items. Hence, we use a binomial regression to replace the beta regression (for a proportion with varying sample size), as an easy approximation which retains the information on the estimation as well as the degree of freedom. A least-squares approach is a simple way of obtaining this (James et al., 2010). Because of the small number of scenarios, we use a Bayesian regression with non-informative priors (Ellison, 1996). In this paper, the data elicited from expert for both designs was fitted by using Bayesian GLM in R using the arm package (Gelman et al., 2016).

\section{Results}

The coefficients and their $95 \%$ credible intervals have been compared across the 3 designs (Figure 2), where the coefficients corresponding to each setting of GLM.

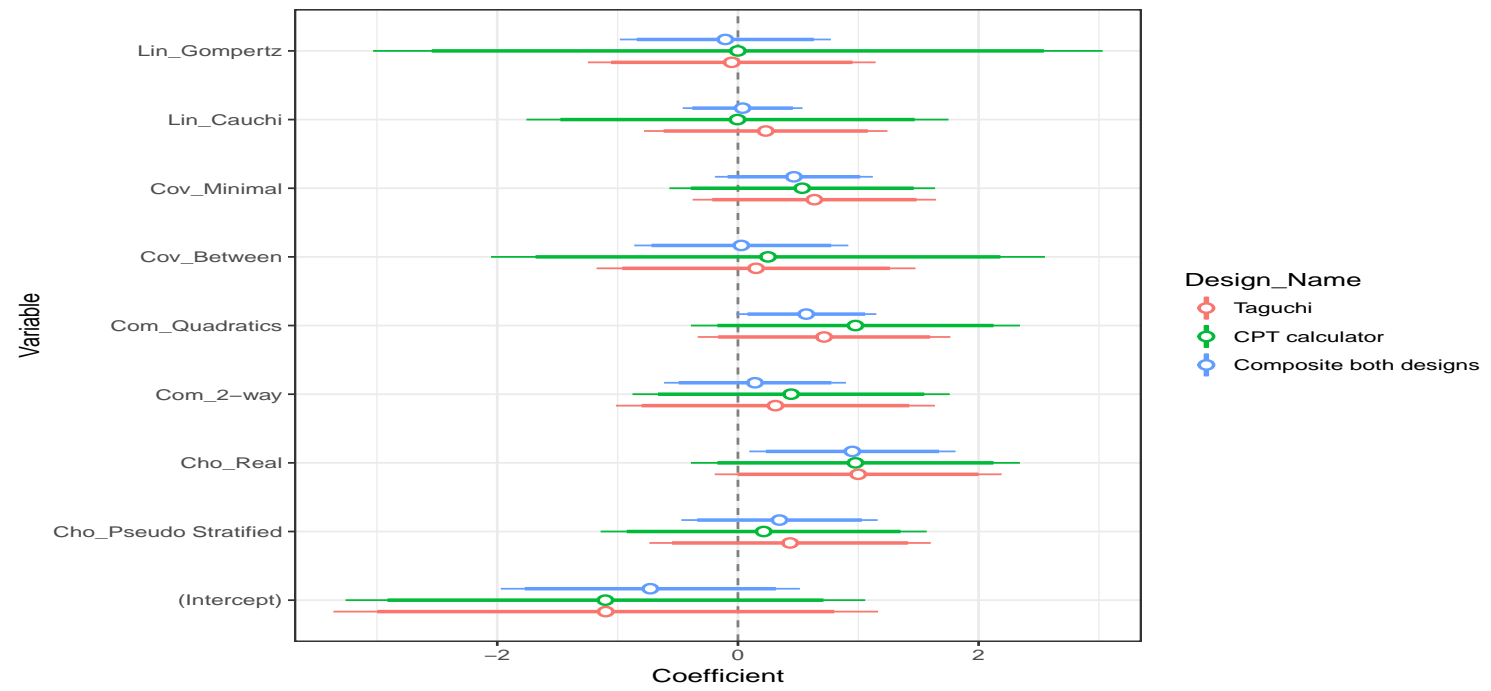

Figure 2. Comparing effects of settings among three designs using estimated coefficients and their $95 \%$ credible intervals from Bayesian GLM.

Using Taguchi OA design to elicit setting-scenarios from the expert, it can be seen that some settings have effect sizes (coefficient value) significantly different from zero. Covariates, complexity of regression and choice of absences in the best case all increase the quality of prediction since their coefficients are larger in magnitude. On the other hand, the "worst" settings of the GLM lower the quality of prediction: link function, complexity of regression, choice of absences and covariates have biggest negative impacts on the quality of predictions.

Using the CPT calculator, similarity the results showed that some settings such as the complexity of regression and choice of absences and covariates in the best case increase the quality of prediction because their coefficients are larger in magnitude. In contrast, the "worst" case settings of the GLM lower the quality of prediction: link function, complexity of regression, choice of absences and covariates have largest negative impacts on the quality of predictions.

When both designs are composited, although the relative order of effect sizes is similar across all three designs, 
we found that it has the smallest effect sizes comparing with Taguchi and CPT calculator designs. Furthermore, the composite design leads to much narrower credible intervals (i.e. decrease ranging from $25 \%$ up to $52 \%$ for the Taguchi design and from $37 \%$ up to $72 \%$ for the CPT calculator design), and hence less uncertainty. Overall, these outputs demonstrated that the largest positive impacts on the quality of prediction are: (1) the choice of real absence data or pseudo-stratified absences, (2) the quadratic complexity of the function applied to covariates and (3) the choice of expert's minimal selected of variables rather than using an extensive set or something in between.

On the other hand, the results in Figure 3 illustrate the comparison among the predictions of three designs compared to values elicited from experts corresponding to all scenarios. It can be seen that there are slight differences between the predictions of each design compared to elicited values. Most Taguchi predictions have matched what the expert said (i.e. nearly 14 out of 17). In contrast, there is a slight difference in CPT calculator predictions compared to elicited values (i.e. nearly 13 out of 17 ). In addition, we noted that the composite design has more scenarios, and so it is easier to obtain more accurate results. Although CPT calculator and Taguchi OA designs have almost the same number of scenarios elicited, the effect sizes estimated for CPT calculator design have wider credible intervals, and hence greater uncertainty, as shown in Figure 2. Therefore, the Taguchi OA design yielded more accurate results than CPT calculator, in this situation.
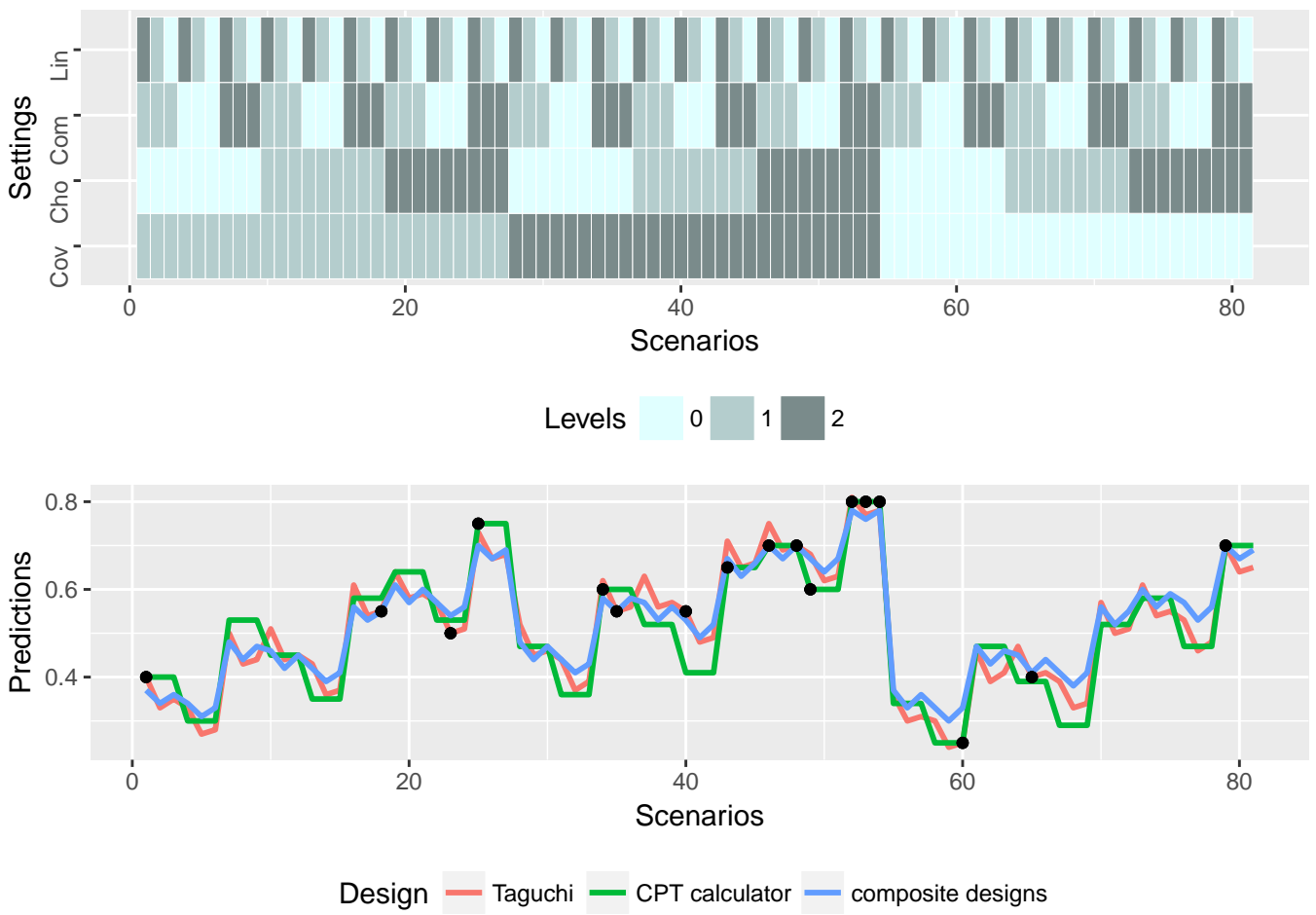

Figure 3. Comparison for each of three designs with elicitation of only a few scenarios (top plot). The predictions of quality of prediction for all scenarios (bottom plot), where the black points refer to the values elicited from experts.

\section{CONCLUSION AND ReCOMmENDATIONS}

In summary, we have used a simple BN to examine how settings of some SDM algorithms (e.g. GLM) influenced the quality of the prediction. We elicited some scenarios (combinations of settings) in the CPT from an expert. Three designs were used to select a few scenarios from the 81 possible: CPT calculator, Taguchi OA and a composite of these designs. This aimed to provide a good coverage of all CPTs as well as obtain accurate predictions. Our results identified the most important settings defining the CPT entries: the choice of real absence data, quadratic complexity of the function and the choice of expert minimal subset of variables each have the largest positive influences on the quality of prediction. In addition, we found that the Taguchi OA design is more efficient than CPT calculator as it has narrower credible intervals which led to more accurate estimation of these influences and predictions. In future work, this paper can be extended to evaluate settings for more SDM algorithms with many experts, and to consider alternative designs for the scenarios based elicitation. 


\section{REFERENCES}

Antony, J. (2003). Design of experiments for engineers and scientists. Elsevier: Oxford.

Box, G. E., J. S. Hunter, and W. G. Hunter (2005). Statistics for experimenters: design, innovation, and discovery. John Wiley \& Sons, Inc.: Hoboken, New Jersey.

Cain, J. (2001). Planning improvements in natural resources management. Centre for Ecology and Hydrology, Wallingford, UK 124, 1-123.

Chen, S. H. and C. A. Pollino (2012). Good practice in Bayesian network modelling. Environmental Modelling \& Software 37, 134-145.

Elith, J. and J. R. Leathwick (2009). Species distribution models: ecological explanation and prediction across space and time. Annual review of ecology, evolution, and systematics 40, 677-697.

Ellison, A. M. (1996). An introduction to Bayesian inference for ecological research and environmental decision-making. Ecological applications 6, 1036-1046.

Fisher, R. A. (1960). The design of experiments. Oliver and Boyd: Edinburgh; London.

Gelman, A., Y.-S. Su, M. Yajima, J. Hill, M. G. Pittau, J. Kerman, T. Zheng, V. Dorie, and M. Y.-S. Su (2016). Package arm. Available from: https://CRAN.R-project.org/package=arm.

Grigore, B., J. Peters, C. Hyde, and K. Stein (2013). Methods to elicit probability distributions from experts: a systematic review of reported practice in health technology assessment. PharmacoEconomics 31(11), 991-1003.

Groemping, U., B. Amarov, H. Xu, and M. U. Groemping (2017). Package 'doe' (Design of Experiments). Available from: http://prof. beuth-hochschule.de/groemping/DoE/.

James, A., S. L. Low Choy, and K. Mengersen (2010). Elicitator: an expert elicitation tool for regression in ecology. Environmental Modelling \& Software 25(1), 129-145.

Jugulum, R., S. Taguchi, et al. (2004). Computer-based Robust Engineering: Essentials for DFSS. ASQ Quality Press: Milwaukee, Wisconsin.

Kacker, R. N., E. S. Lagergren, and J. J. Filliben (1991). Taguchi's Orthogonal Arrays Are Classical Designs of Experiments. Journal of research of the National Institute of Standards and Technology 96(5), 577-591.

Korb, K. B. and A. E. Nicholson (2010). Bayesian artificial intelligence. CRC press: London, UK.

Low Choy, S., A. James, J. Murray, and K. Mengersen (2012). Elicitator: a user-friendly, interactive tool to support scenario-based elicitation of expert knowledge. In Expert Knowledge and Its Application in Landscape Ecology, Chapter 3, pp. 39-67. Springer: New York.

Low Choy, S., J. Murray, A. James, and K. L. Mengersen (2010). Indirect elicitation from ecological experts: from methods and software to habitat modelling and rock-wallabies. The Oxford Handbook Of Applied Bayesian Analysis, 511-544.

Low Choy, S., R. O'Leary, and K. Mengersen (2009). Elicitation by design in ecology: using expert opinion to inform priors for Bayesian statistical models. Ecology 90(1), 265-277.

Marcot, B. G., J. D. Steventon, G. D. Sutherland, and R. K. McCann (2006). Guidelines for developing and updating Bayesian belief networks applied to ecological modeling and conservation. Canadian Journal of Forest Research 36(12), 3063-3074.

Pike, W. A. (2004). Modeling drinking water quality violations with Bayesian Networks. American Water Resources Association 40(6), 1563 - 1578.

Reiss, H., S. Cunze, K. König, H. Neumann, and I. Kröncke (2011). Species distribution modelling of marine benthos: a north sea case study. Marine Ecology Progress Series 442, 71-86.

Speirs-Bridge, A., F. Fidler, M. McBride, L. Flander, G. Cumming, and M. Burgman (2010). Reducing overconfidence in the interval judgments of experts. Risk Analysis 30(3), 512-523.

Taguchi, G. and S. Konishi (1987). Taguchi Methods: Orthogonal Arrays and Linear Graphs-Tools for Quality Engineering. American Supplier Institute: Dearborn, Mich. 